\title{
Genomic analysis of invasion-metastasis-related factors in pancreatic cancer cells
}

\author{
XIAODONG TAN $^{1^{*}}$, LEI ZHOU ${ }^{1 *}$, WEI WANG ${ }^{1}$, BAOSHENG WANG $^{1}$, \\ HIROSHI EGAMI ${ }^{2}$, HIDEO BABA ${ }^{3}$ and XIANWEI DAI ${ }^{1}$

\begin{abstract}
${ }^{1}$ Department of Hepato-pancreato-biliary Tumor Surgery, China Medical University Shengjing Hospital, Shenyang, P.R. China; ${ }^{2}$ Department of Surgery, Ugatake Hospital; ${ }^{3}$ Department of Gastroenterological Surgery,
\end{abstract} \\ Graduate School of Medical Sciences, Kumamoto University, Kumamoto, Japan
}

Received August 21, 2009; Accepted October 19, 2009

DOI: 10.3892/etm_00000034

\begin{abstract}
Pancreatic cancer is known to be an extremely lethal neoplasm, one of the reasons being that pancreatic cancer itself has an extremely high potential of invasionmetastasis. In our previous study, two pancreatic cancer cell lines with a different potential for invasion-metastasis, PC-1 with a low potential and PC-1.0 with a high potential of invasion-metastasis after intrapancreatic transplantation, were established in a Syrian golden hamster. To determine the invasion-metastasis-related factors, a cDNA microarray that represented a set of 27,000 genes was hybridized with a labeled cDNA probe and screened for molecular profiling analysis. Furthermore, Gene Ontology and Pathway differential expression of candidate genes was further validated using RT-PCR. One hundred and forty-one differentially expressed genes ( $>3.0$-fold change) were identified in the present study, including 46 up-regulated genes (e.g., nup107, tjp-2 and MMP-13) and 95 down-regulated genes (e.g., Spc21, plau and CD44) in the PC-1.0 cells. Our present results suggest that a highly organized and structured process of tumor invasionmetastasis exists in the pancreas. Analysis of gene expression profiles by cDNA microarray provides useful information for clarifying the mechanism underlying this invasion and metastasis. Furthermore, the identification of invasionmetastasis-specific genes may allow us to develop new therapeutic and diagnostic targets for the invasion-metastasis of pancreatic cancer.
\end{abstract}

Correspondence to: Dr Xianwei Dai, Department of Hepatopancreato-biliary Tumor Surgery, China Medical University Shengjing Hospital, No. 36 Sanhao Street, Heping District, Shenyang 110004, P.R. China

E-mail: tanxd@hotmail.com

*Contributed equally

Key words: pancreatic cancer, invasion, metastasis, cDNA microarray

\section{Introduction}

One of the most lethal features of pancreatic cancer is its apparent capacity for early invasion and metastasis to the liver and other organs. Apart from surgery, there is no effective therapy and even resected patients usually die within one year postoperatively. Reasons for the poor prognosis include the occurrence of local recurrences and/or distant metastasis after surgery. However, to date, the cellular and molecular mechanisms of the invasion-metastasis of pancreatic cancer remain unclear. Detection of the factors related to the differences in potential for invasion and metastasis of cancer cells could provide useful information for the development of new therapeutic methods to prevent the invasion and metastasis of pancreatic cancer.

To investigate the mechanisms of invasion-metastasis of pancreatic cancer, two hamster pancreatic cancer cell lines with a different potential for invasion and metastasis, PC-1 with a low potential and PC-1.0 with a high potential after intrapancreatic transplantation, were established from a pancreatic ductal carcinoma induced by $\mathrm{N}$-nitrosobis (2-oxopropyl) amine (BOP) in a Syrian golden hamster in our previous investigation $(1,2)$.

cDNA microarray is a new emerging technique in the post genomic era. Large-scale analysis of gene expression with cDNA microarray allows us to evaluate the gene expression profiles of hundreds to tens of thousands of genes in a single experiment (3). Therefore, the cDNA microarray is a promising tool to provide new insight into the mechanisms of cancer invasion and metastasis.

In the present study, we analyzed alteration in the invasionmetastasis-related gene expression patterns of 27,000 genes in highly invasive and metastatic pancreatic cancer cells (PC-1.0) in comparison to weakly invasive and metastatic pancreatic cancer cells (PC-1) utilizing powerful cDNA microarray technology.

\section{Materials and methods}

Cell lines and cell culture. Two hamster pancreatic cancer cell lines, weakly invasive and metastatic cells (PC-1) and highly invasive and metastatic cells (PC-1.0) were used. The 
PC-1 cell line was established from pancreatic ductal/ductular adenocarcinomas induced by BOP in a Syrian golden hamster (1). The PC-1.0 cell line was established from a subcutaneous tumor produced after inoculation of PC-1 cells (2). In vitro, PC-1 cells grow mainly as island-like cell colonies, whereas PC-1.0 cells exhibit the growth pattern of single cells. In vivo, local expansion of PC-1 cells and local invasion of PC-1.0 cells are observed $(1,2)$.

The PC-1 and PC-1.0 cells were incubated in RPMI-1640 (Gibco-BRL, Grand Island, NY, USA), supplemented with $10 \%$ fetal bovine serum (Bioserum, Victoria, Australia), $100 \mathrm{U} / \mathrm{ml}$ penicillin $\mathrm{G}$ and $100 \mu \mathrm{g} / \mathrm{ml}$ streptomycin at $37^{\circ} \mathrm{C}$ in a humidified atmosphere of $5 \% \mathrm{CO}_{2} / 95 \%$ air.

Preparation of total RNA. Total RNA of the PC-1.0 and PC-1 cells was extracted using the TRIzol reagent according to the manufacturer's instructions (Invitrogen). After TRIzol purification, RNA was further purified with RNeasy mini spin column kit (Qiagen, Valencia, CA, USA). The concentration and qualify of the RNA were assessed via spectrophotometry and agarose gel electrophoresis.

cDNA microarray and statistical analysis of data. Preparation of fluorescent dye-labeled DNA and hybridizations was performed according to the protocol of the reagent/kit manufacturers and previously reported methods (4). Briefly, RNA was reverse-transcribed into cDNA with Oligo(dT)15 (Promega) as primer and Superscript II choice for cDNA synthesis (Invitrogen) and subsequently labeled in red (Cy5) or in green (Cy3) (Amersham Pharmacia Biotech). Cy5- and Cy3-labeled cDNA was purified with a PCR purification kit (Qiagen). DNA was mixed with $30 \mu$ l hybridization solution prior to loading onto a rat gene microarray (Capitalbio Inc., Beijing, P.R. China) which included 27,000 transcripts (Oligo library, Rat Genome version 3.0.5; Qiagen). Arrays were hybridized at $42^{\circ} \mathrm{C}$ overnight. The experiments were performed twice with reverse dye-labeled cDNA.

The microarray plates were scanned by LuxScan 10KA dual pathways laser scanner (Capitalbio), and images wereanalyzed through GenePix Pro 4.0 image analysis software (Axon Instruments Co.). Genes were considered to be differentially expressed, integrated ratio of two experiments, at a change in increase $(>3.00)$ or decrease $(<0.33)$ in the ratio of expression levels between PC-1.0 and PC-1 cells.

Statistical analysis was carried out with the t-test, and the expression of a given gene was considered changed when the difference between means was significant $(\mathrm{P}<0.01)$.

Reverse transcriptase-polymerase chain reaction (RT-PCR). Total RNA was isolated from PC-1.0 and PC-1 cells, and an aliquot of $1 \mu \mathrm{g}$ of total RNA from each sample was reversetranscribed to cDNA using the SuperScript II kit (Life Technologies, Inc.) as previously described (5). The primers used for PCR amplification in this study are listed in Table I. Amplification was run for 30 cycles at $95^{\circ} \mathrm{C}$ for $5 \mathrm{~min}, 95^{\circ} \mathrm{C}$ for $40 \mathrm{sec}, 55^{\circ} \mathrm{C}$ for $30 \mathrm{sec}, 72^{\circ} \mathrm{C}$ for $1 \mathrm{~min}$ and finally extended at $72^{\circ} \mathrm{C}$ for $7 \mathrm{~min}$.

Gene Ontology and Pathway analysis of differentially expressed genes. Using the Gene Ontology tool from http://
Table I. Primers used for the RT-PCR of PC-1.0 and PC-1 cells.

\begin{tabular}{llc}
\hline $\begin{array}{l}\text { Gene } \\
\text { name }\end{array}$ & \multicolumn{1}{c}{$\begin{array}{c}\text { Primer } \\
\text { sequence }\end{array}$} & $\begin{array}{c}\text { Product } \\
\text { size (bp) }\end{array}$ \\
\hline Actin & $\begin{array}{l}\text { F: GTGGGGCGCCCCAGGCACCA } \\
\text { R: CTCCTTAAGTCACGCACGATTCC }\end{array}$ & 664 \\
nup107 & F: GACAGAAGAGGCACAACGAC & 309 \\
& R: ACCAGACTGTCCACCATCAC & \\
tjp2 & F: GCAGAGCGAACGAAGAGTATGG & 245 \\
& R: TGACGGGATGTTGATGAGGGT & \\
MMP-13 & F: CAGTCTTTCTTCGGCTTAG & 496 \\
& R: CAGGGTCCTTGGAGTGGTC & \\
Spc21 & $\begin{array}{l}\text { F: GTGGTGCTGAGTGGCAGTAT } \\
\text { R: CCAGTTCTGGCCTTCTTTGT }\end{array}$ & 246 \\
& $\begin{array}{l}\text { F: AGAATTCACCACCATCGAGA } \\
\text { Rlau }\end{array}$ & 474 \\
CD44 & F: ATCAGCTTCACAACAGTCAT & \\
& R: AACTGCAATGCAAACTGCAAG & \\
\hline
\end{tabular}

www.pantherdb.org, the differentially expressed genes were automatically assembled to categories of Biological process, Molecular function and Cellular component. Biologically related networks were automatically assembled from identified genes on microarrays by the BioRag (http://www.biorag.org), which enables the analysis of pathways among interested genes according to Kegg (http://www.genome.ad.jp/kegg) or GenMAPP (http://www.genmapp.org). The Fisher's exact test was performed to detect the significantly regulated gene and pathway, A P-value $<0.01$ was considered significantly overrepresented.

\section{Results}

Differentially expressed genes identified by cDNA microarray in the highly (PC-1.0) and weakly (PC-1) invasive and metastatic pancreatic cancer cells. To clarify the differentially expressed genes between highly (PC-1.0) and weakly (PC-1) invasive and metastatic cells, the expression level for each gene in the two pancreatic cancer cell lines was compared. Of the 27,000 genes analyzed through microarray experiments, a total of 141 genes revealed differential expression using a fold ratio $>3$ as the criteria for cut-off. Of the 141 genes, the expression of 46 genes (32.6\%) was markedly increased in the highly invasive and metastatic cells (PC-1.0) as compared with the weakly invasive and metastatic cells (PC-1) (Table II). On the other hand, the expression of 95 genes (67.4\%) was significantly decreased in the highly invasive and metastatic cells (PC-1.0) as compared with the weakly invasive and metastatic cells (PC-1) (Table III). The ratio represented the expression value in PC-1.0 cells compared with the expression level in $\mathrm{PC}-1$ cells.

Validation of selected genes with RT-PCR. To verify the reliability of the microarray data, we selected three up-regulated 
Table II. Genes up-regulated in highly invasive and metastatic cells (PC-1.0) compared with weakly invasive and metastatic cells (PC-1).

\begin{tabular}{|c|c|c|c|c|}
\hline Gene name & Gene ID & Gene symbol & Description & Ratio \\
\hline Mlp & ENSRNOG00000009113 & NM_030862 & MARCKS-like protein & 71.9931 \\
\hline Aldr1 & ENSRNOG00000009513 & ALDR_RAT & Aldehyde reductase 1 & 33.6872 \\
\hline MMP-13 & ENSRNOG00000008478 & MM13_RAT & Matrix metallopeptidase 13 & 30.0071 \\
\hline MMP-12 & ENSRNOG00000008993 & MM03_RAT & Matrix metallopeptidase 12 & 26.2124 \\
\hline Col5a2 & ENSRNOG00000003736 & O70598 & Collagen, type V, $\alpha 2$ & 20.8255 \\
\hline Tnni2 & ENSRNOG00000020276 & TRIF_RAT & Troponin 1 , type 2 & 20.5716 \\
\hline Tjp2 & ENSRNOG00000015030 & P70625 & Tjp2 protein & 20.5690 \\
\hline MMP-3 & ENSRNOG00000008993 & MM03_RAT & Matrix metallopeptidase 3 & 20.5668 \\
\hline Snrpn & ENSRNOG00000022595 & NM_130738 & Small nuclear ribonucleoprotein $\mathrm{N}$ & 17.1193 \\
\hline Syt8 & ENSRNOG00000020245 & NM_053325 & Synaptotagmin 8 & 15.8243 \\
\hline S100a5 & ENSRNOG00000011748 & S105_MOUSE & S100 calcium binding protein A5 & 11.6390 \\
\hline Ndrg2 & ENSRNOG00000010389 & NM_133583 & $\mathrm{N}$-myc downstream regulated gene 2 & 11.4480 \\
\hline MMP-10 & ENSRNOG00000008993 & MM03_RAT & Matrix metallopeptidase 10 & 10.7218 \\
\hline $\mathrm{Tf}$ & ENSRNOG00000009434 & TRFE_RAT & Transferrin & 8.7425 \\
\hline Anxa6 & ENSRNOG00000010668 & ANX6_RAT & Annexin A6 & 6.5978 \\
\hline Nup107 & ENSRNOG00000006541 & N107_RAT & Nucleoporin 107 & 6.5895 \\
\hline Spnb3 & ENSRNOG00000019564 & SPCP_RAT & $\beta$-spectrin 3 & 6.3763 \\
\hline Cdk4 & ENSRNOG00000025602 & CDK4_RAT & Cyclin-dependent kinase 4 & 6.2099 \\
\hline Fap & ENSRNOG00000005679 & NM_138850 & Fibroblast activation protein & 5.7157 \\
\hline Eno3 & ENSRNOG00000004078 & ENOB_RAT & Enolase $3, \beta$ & 5.6429 \\
\hline
\end{tabular}

The complete data of the cDNA microarray analysis is available upon request.

Table III. Genes down-regulated in highly invasive and metastatic cells (PC-1.0) compared with weakly invasive and metastatic cells (PC-1).

\begin{tabular}{lllll}
\hline Gene name & \multicolumn{1}{c}{ Gene ID } & Gene symbol & \multicolumn{1}{c}{ Description } & Ratio \\
\hline App & ENSRNOG00000001546 & A4_RAT & Amyloid $\beta$ (A4) precursor protein & 0.1286 \\
Co19a1 & ENSRNOG00000012920 & CA19_RAT & Procollagen, type IX, $\alpha$ 1 & 0.1269 \\
CD44 & ENSRNOG00000013562 & CD44_RAT & CD44 antigen & 0.1205 \\
Serpinh1 & ENSRNOG00000016831 & HS47_RAT & Serine proteinase inhibitor 1, clade H & 0.1190 \\
Tcf4 & ENSRNOG00000012405 & ITF2_RAT & Transcription factor 4 & 0.1039 \\
Chn2 & ENSRNOG00000009411 & CHIO_RAT & Chimerin (chimaerin) 2 & 0.0913 \\
Plau & ENSRNOG00000010516 & UROK_RAT & Plasminogen activator, urokinase & 0.0895 \\
Sphk1 & ENSRNOG00000010626 & NM_133386 & Sphingosine kinase 1 & 0.0882 \\
Apom & ENSRNOG00000000850 & APOM_RAT & Apolipoprotein M & 0.0850 \\
Psmb8 & ENSRNOG00000000456 & PSB8_RAT & Proteosome subunit, $\beta$ type 8 & 0.0844 \\
Ldhb & ENSRNOG00000013000 & LDHB_RAT & Lactate dehydrogenase B & 0.0737 \\
Spc21 & ENSRNOG00000017036 & SPC3_RAT & Microsomal signal peptidase 21 kDa subunit & 0.0712 \\
Klf4 & ENSRNOG00000016299 & NM_053713 & Kruppel-like factor 4 & 0.0669 \\
Cntn4 & ENSRNOG00000005652 & NM_053746 & Contactin 4 & 0.0508 \\
Ephx1 & ENSRNOG00000003515 & HYEP_RAT & Epoxide hydrolase 1 & 0.0493 \\
Serpinb2 & ENSRNOG00000002460 & PAI2_RAT & Plasminogen activator inhibitor 2 & 0.0465 \\
Pmp22 & ENSRNOG00000003338 & PM22_RAT & Peripheral myelin protein 22 & 0.0337 \\
Pde1c & ENSRNOG00000012337 & CN1C_RAT & Phosphodiesterase 1C & 0.0322 \\
Ngfrap1 & ENSRNOG00000012646 & NM_053401 & Nerve growth factor receptor & 0.0218 \\
Hspb1 & ENSRNOG00000023546 & HS27_RAT & Heat shock 27 kDa protein 1 & \\
\hline
\end{tabular}

The complete data of the cDNA microarray analysis is available upon request. 
Table IV. Gene Ontology analysis - Molecular function.

\begin{tabular}{lcccc}
\hline GO Term & Total & P-value & Gene & Input symbol \\
GO:0004852 uroporphyrinogen-III synthase activity & 1 & 0.0043 & Uros & Rn30016380 \\
GO:0000900 translation repressor activity & 1 & 0.0043 & Purb & Rn30006362 \\
GO:0005131 growth hormone receptor binding & 1 & 0.0086 & Socs2 & R002975_01 \\
GO:0030161 calpain inhibitor activity & 1 & 0.0086 & Cast & R001975_01 \\
GO:0046980 tapasin binding & 1 & 0.0086 & Tap2 & Rn30000347 \\
GO:0004308 exo- $\alpha$-sialidase activity & 1 & 0.0129 & Neu1 & R003273_01 \\
GO:0005518 collagen binding & 1 & 0.0172 & Serpinh1 & R003232_01 \\
GO:0008538 proteasome activator activity & 1 & 0.0172 & Psme1 & Rn30017518 \\
GO:0008243 plasminogen activator activity & 1 & 0.0214 & Plau & Rn30009672 \\
GO:0019838 growth factor binding & 1 & 0.1738 & axl & Rn30019093 \\
\hline
\end{tabular}

Table V. Gene Ontology analysis - Biological process.

\begin{tabular}{|c|c|c|c|c|}
\hline GO Term & Total & P-value & Gene & Input symbol \\
\hline GO:0015914 phospholipid transport & 1 & $1.10 \mathrm{E}-4$ & Plscr1 & Rn30007316 \\
\hline GO:0006983 ER overload response & 1 & $1.83 \mathrm{E}-4$ & Ddit3 & Rn30006089 \\
\hline GO:0006955 immune response & 4 & 0.0068 & $\begin{array}{c}\text { Tap2 } \\
\text { Ada } \\
\text { Psme1 } \\
\text { Plscr1 }\end{array}$ & $\begin{array}{l}\text { Rn30000347 } \\
\text { R004405_01 } \\
\text { Rn30017518 } \\
\text { Rn30007316 }\end{array}$ \\
\hline GO:0007034 vacuolar transport & 1 & 0.0086 & Vps $26 a$ & Rn30000282 \\
\hline GO:0031100 organ regeneration & 1 & 0.0172 & axl & Rn30019093 \\
\hline GO:0007520 myoblast fusion & 1 & 0.0172 & Cast & R001975_01 \\
\hline GO:0009968 negative regulation of signal transduction & 2 & 0.0183 & $\begin{array}{l}\text { Socs } 2 \\
\text { Rgs } 10\end{array}$ & $\begin{array}{l}\text { R002975_01 } \\
\text { Rn30018565 }\end{array}$ \\
\hline GO:0050892 intestinal absorption & 1 & 0.0257 & $\mathrm{Vdr}$ & Rn30007787 \\
\hline GO:0001558 regulation of cell growth & 1 & 0.0331 & Igfbp6 & Rn30010107 \\
\hline GO:0035023 regulation of Rho protein signal transduction & 1 & 0.0382 & Net1 & Rn30016337 \\
\hline
\end{tabular}

$\mathbf{A}$

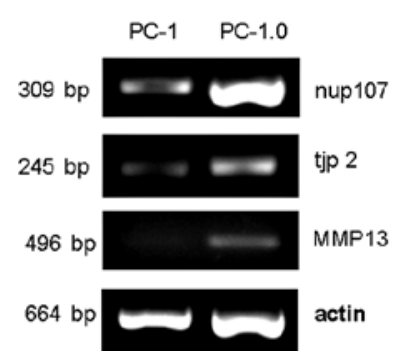

Figure 1. Validation of cDNA microarray data by RT-PCR. (A) The expression levels of up-regulated genes (nup107, tjp2 and MMP13) in highly (PC-1.0) and weakly (PC-1) invasive and metastatic pancreatic cancer cells. (B) The expression levels of down-regulated genes (Spc21, plau and CD44) in highly (PC-1.0) and weakly (PC-1) invasive and metastatic pancreatic cancer cells.

genes (nup107, tjp2 and MMP13) and three down-regulated genes (Spc21, plau and CD44) to measure their expression levels by RT-PCR. The results were very similar to the cDNA microarray data on these genes and supported the reliability of our expression data (Fig. 1).

Gene Ontology and Pathway analysis of differentially expressed genes. Gene Ontology (GO) and Pathway analysis was applied in order to generate groups of genes that belong to similar biological processes correlated with invasion and metastasis of pancreatic cancer cells.

The differentially expressed genes between highly (PC-1.0) and weakly (PC-1) invasive and metastatic cells were summarized in Molecular function, Biological process and Cellular component, respectively. These are the three types of categories of $\mathrm{GO}$ analysis. The ten most correlated (the lowest P-value) GO categories of Molecular function, Biological Process and Cellular Component are presented in Tables IV, $\mathrm{V}$ and VI, respectively.

In addition, Pathway analysis of differentially expressed genes was also applied using the public database (Kegg and GenMAPP). The ten most correlated pathways obtained from 
Table VI. Gene Ontology analysis - Cellular component.

\begin{tabular}{lcccc}
\hline Go Term & Total & P-value & Gene & Input symbol \\
\hline GO:0005923 tight junction & 1 & 0.0156 & Tjp2 & Rn30013789 \\
GO:0005788 endoplasmic reticulum lumen & 1 & 0.0340 & Tap2 & Rn30000347 \\
GO:0030904 retromer complex & 1 & 0.0043 & Vps26a & Rn30000282 \\
GO:0008537 proteasome activator complex & 1 & 0.0172 & Psme1 & Rn30017518 \\
GO:0005662 DNA replication factor A complex & 1 & 0.0040 & Purb & Rn30006362 \\
GO:0042589 zymogen granule membrane & 1 & 0.0214 & Scamp1 & R004473_01 \\
GO:0005793 ER-Golgi intermediate compartment & 1 & 0.0506 & Serpinh1 & R003232_01 \\
GO:0005905 coated pit & 1 & 0.0949 & Vldlr & Rn30025704 \\
GO:0019717 synaptosome & 1 & 0.0382 & Vamp3 & Rn30017017 \\
GO:0016020 membrane & 1 & 0.6022 & axl & Rn30019093 \\
\hline
\end{tabular}

Table VII. Pathway analysis - Kegg.

\begin{tabular}{lcc}
\hline Pathway name & Total & P-value \\
\hline $\begin{array}{l}\text { Pentose and glucuronate } \\
\text { interconversions }\end{array}$ & 4 & 0.0000 \\
Antigen processing and presentation & 8 & $2.7 \mathrm{E}-5$ \\
$\begin{array}{l}\text { Starch and sucrose metabolism } \\
\text { Porphyrin and chlorophyll metabolism }\end{array}$ & 3 & $3.15 \mathrm{E}-4$ \\
Sphingolipid metabolism & 5 & $3.65 \mathrm{E}-4$ \\
Fructose and mannose metabolism & 5 & $3.65 \mathrm{E}-4$ \\
$\begin{array}{l}\text { Phenylalanine, tyrosine and } \\
\text { tryptophan biosynthesis }\end{array}$ & 3 & $0.51 \mathrm{E}-4$ \\
Type I diabetes mellitus & & 0.0011 \\
$\begin{array}{l}\text { Metabolism of xenobiotics P450 } \\
\text { by cytochrome }\end{array}$ & 4 & 0.0028 \\
SNARE interactions in vesicular transport & 4 & 0.0028 \\
\hline
\end{tabular}

Table VIII. Pathway analysis - GenMAPP.

\begin{tabular}{lcl}
\hline Pathway name & Total & P-value \\
\hline GTP binding & 23 & $2.0 \mathrm{E}-6$ \\
Guanyl nucleotide binding & 23 & $3.0 \mathrm{E}-6$ \\
Cytosol & 15 & $4.0 \mathrm{E}-6$ \\
Endoplasmic reticulum & 23 & $7.0 \mathrm{E}-6$ \\
Binding & 21 & $8.0 \mathrm{E}-6$ \\
Cytoplasm & 21 & $7.30 \mathrm{E}-5$ \\
Electron transport & 20 & $2.11 \mathrm{E}-4$ \\
Magnesium ion binding & 11 & $2.74 \mathrm{E}-4$ \\
Protein folding & 14 & $3.52 \mathrm{E}-4$ \\
RNA binding & 20 & $3.69 \mathrm{E}-4$ \\
Metabolism & 21 & $3.73 \mathrm{E}-4$ \\
\hline
\end{tabular}

the Kegg and GenMAPP are listed in Tables VII and VIII, respectively.

The complete data of the GO and Pathway analysis is available upon request.

\section{Discussion}

To date, there have been some reports regarding the molecular mechanisms involved in the development of pancreatic cancer, including some reports utilizing cDNA microarray $(6,7)$. However, thus far, most of these cDNA microarray studies have focused on the differences between pancreatic cancer tissue and normal tissue (8); few studies have investigated the mechanism of invasion and metastasis in pancreatic cancer cells using highly and weakly invasive and metastatic pancreatic cancer cell lines. Yet, these tissue samples have considerable disadvantages. They are highly complex and are usually composed of several different cell types and extracellular matrices; for example, non-neoplastic pancreatic tissue includes ductal and acinar cells, various neuroendocrine cells and mesenchymal cells. Thus, one has to be aware that using samples of tissue homogenates does not simply mean a comparison of neoplastic vs. non-neoplastic epithelial cells, but a complex mixture of genes of diverse origin, some of them deriving from epithelial cells. In contrast, one advantage of using cancer cell lines is that pure tumor cells are tested without any contamination from surrounding stromal elements.

In particular, the highly (PC-1.0) and weakly (PC-1) invasive and metastatic pancreatic cancer cell lines, which are established from the experimental pancreatic cancer model in our previous study $(1,2)$, show an obviously different potential for invasion and metastasis $(9,10)$. Therefore, this cell line model is suitable for the investigation of invasion-metastasisrelated specific factors in pancreatic cancer.

In the present study, using cDNA microarray analysis, we found that a total of 141 genes were differentially expressed between the PC-1.0 and PC-1 cells, including 46 up-regulated genes and 95 down-regulated genes. We selected several differentially expressed genes (nup107, tjp-2, MMP-13, Spc21, plau and CD44) for validation by RT-PCR. The results of RT-PCR were in accordance with those of the cDNA microarray analysis. In addition, several of the identified genes (i.e., MMP-13, plau and CD44) have been previously reported to be correlated with invasion and metastasis (11-13), and the other differentially expressed genes (i.e., nup107, tjp-2 and Spc21) have not been reported to be associated with the invasion-metastasis of pancreatic cancer. 
Of the identified genes not previously reported to be associated with the invasion-metastasis of pancreatic cancer, Nup107 is a critical component of the nucleoporin 107-160 subcomplex, which is the key building block of the nuclearpore complex (NPC). From yeast to humans, the function of NPC is the regulation of nuclear import and export (14). The Nup107-160 complex thus additionally offers an attractive point for regulation of nuclear pore complex assembly (15). Although nup107 has been identified from the comparison of gene expression in highly and weakly invasive and metastatic pancreatic cancer cells in the present study, the molecular mechanism of involvement of nup107 in the invasion-metastasis of pancreatic cancer needs to be further tested and assessed.

Several studies have demonstrated that tight junction proteins (TJPs) associate with each other and directly and/ or indirectly to actin filaments (16) and also recruit factors involved in signal transduction and the regulation of proliferation and differentiation (17). The zonula occludens (ZO) protein is one of the tight junction proteins and belongs to the membrane associated guanylate kinase-like (MAGUK) protein family. It includes three members, TJP1/ZO-1, TJP2/ $\mathrm{ZO}-2$ and TJP3/ZO-3 (18). mRNA levels of ZO-2 were found to be elevated in tumor tissues compared with controls using quantitative PCR. Moreover, ZO-2 exhibits a 23-amino acid truncation at the $\mathrm{N}$-terminus, which may play a role in limiting tumor development in pancreatic cells. In another investigation, ZO-2 was found to be associated with the progression of breast cancer (19).

Moreover, Spc21 was identified as a down-regulated gene in this study, suggesting that dysregulation of this gene is likely to be associated with the invasion and metastasis of pancreatic cancer cells. Fish and ISH analysis for this gene demonstrated a significant correlation between genetic deletion and corresponding mRNA down-regulation, raising the possibility that the Spc21 gene may play a putative role as a tumor suppressor (20). However, little is known about the biological role of this gene, although it belongs to the peptidase S26B family and functions as part of the signal peptidase complex (20).

In conclusion, our results suggest that a highly organized and structured process of invasion and metastasis exists in the pancreas. Analysis of gene expression profiles by cDNA microarray can provide useful information for clarifying the mechanism underlying the invasion and metastasis of pancreatic cancer cells. Furthermore, the identification of invasion-metastasis-specific genes may allow us to develop new therapeutic and diagnostic targets for the invasion-metastasis of pancreatic cancer.

\section{Acknowledgements}

This study was supported by a grant-in-aid from the China Postdoctoral Science Foundation (no. 20060390302). We thank Professor Hideo Baba for the kind gift of PC-1 and PC-1.0 cell lines.

\section{References}

1. Egami H, Takiyama Y, Cano M, Houser WH and Pour PM: Establishment of hamster pancreatic ductal carcinoma cell line (PC-1) producing blood group-related antigens. Carcinogenesis 10: 861-869, 1989.

2. Egami H, Tomioka T, Tempero M, Kay D and Pour PM: Development of intrapancreatic transplantable model of pancreatic duct adenocarcinoma in Syrian golden hamsters. Am J Pathol 138: 557-561, 1991.

3. Goggins M: Identifying molecular markers for the early detection of pancreatic neoplasia. Semin Oncol 34: 303-310, 2007.

4. Shi YH, Zhu SW, Mao XZ, et al: Transcriptome profiling, molecular biological and physiological studies reveal a major role for ethylene in cotton fiber cell elongation. Plant Cell 18: 651-664, 2006.

5. Tan X, Egami H, Kamohara $\mathrm{H}$, et al: Involvement of the mitogen-activated protein kinase kinase 2 in the induction of cell dissociation in pancreatic cancer. Int J Oncol 24: 65-73, 2004

6. Duerr EM, Mizukami Y, Ng A, et al: Defining molecular classifications and targets in gastroenteropancreatic neuroendocrine tumors through DNA microarray analysis. Endocr Relat Cancer 15: 243-256, 2008.

7. Jones S, Zhang X, Parsons DW, et al: Core signaling pathways in human pancreatic cancers revealed by global genomic analysis. Science 321: 1801-1806, 2008.

8. Capurso G, Lattimore S, Crnogorac-Jurcevic T, et al: Gene expression profiles of progressive pancreatic endocrine tumours and their liver metastases reveal potential novel markers and therapeutic targets. Endocr Relat Cancer 13: 41-58, 2006.

9. Pour PM, Egami H and Takiyama Y: Patterns of growth and metastases of induced pancreatic cancer in relation to the prognosis and its clinical implication. Gastroenterology 100: 529-536, 1991.

10. Kurizaki T, Egami H, Hirota M, et al: Characterization of cancer cell dissociation factor in a highly invasive pancreatic cancer cell line. Cancer 75: 1554-1561, 1995.

11. Morgia G, Falsaperla M, Malaponte G, et al: Matrix metalloproteinases as diagnostic (MMP-13) and prognostic (MMP-2, MMP-9) markers of prostate cancer. Urol Res 33: 44-50, 2004.

12. Iiizumi M, Liu W, Pai SK, Furuta E and Watabe K: Drug development against metastasis-related genes and their pathways: a rationale for cancer therapy. Biochim Biophys Acta 1786: 87-104, 2008.

13. Klingbeil P and Marhaba R: CD44 variant isoforms promote metastasis formation by a tumor cell-matrix cross-talk that supports adhesion and apoptosis resistance. Mol Cancer Res 7: 168-179, 2009.

14. Schuldt A: Nuclear pore assembly: locating the linchpin. Nat Cell Biol 5: 497, 2003.

15. Harel A, Orjalo AV, Vincent $\mathrm{T}$, et al: Removal of a single pore subcomplex results in vertebrate nuclei devoid of nuclear pores. Mol Cell 11: 853-864, 2003.

16. Utepbergenov DI, Fanning AS and Anderson JM: Dimerization of the scaffolding protein ZO-1 through the second PDZ domain. J Biol Chem 281: 24671-24677, 2006.

17. Matter $\mathrm{K}$ and Balda MS: Functional analysis of tight junctions. Methods 30: 228-234, 2003.

18. Sato N, Fukushima N, Maitra A, et al: Discovery of novel targets for aberrant methylation in pancreatic carcinoma using highthroughput microarrays. Cancer Res 63: 3735-3742, 2003.

19. Martin TA, Watkins G, Mansel RE and Jiang WG: Loss of tight junction plaque molecules in breast cancer tissues is associated with a poor prognosis in patients with breast cancer. Eur J Cancer 40: 2717-2725, 2004

20. Harada T, Baril P, Gangeswaran R, et al: Identification of genetic alterations in pancreatic cancer by the combined use of tissue microdissection and array-based comparative genomic hybridization. Br J Cancer 96: 373-382, 2007. 